\title{
Melhora no Consumo Máximo de Oxigênio e na Ventilação após Tratamento com Sacubitril-Valsartana
}

\author{
Maximal Oxygen Uptake and Ventilation Improvement Following Sacubitril-Valsartan Therapy
}

António Valentim Gonçalves, ${ }^{10}$ Tiago Pereira-da-Silva, ${ }^{1}$ Ana Galrinho, ${ }^{1}$ Pedro Rio, ${ }^{1}$ Rui Soares, ${ }^{1}$ Joana Feliciano, ${ }^{1}$ Rita Ilhão Moreira, ${ }^{1}$ Sofia Silva, ${ }^{1}$ Sandra Alves, ${ }^{1}$ Eunice Capilé, ${ }^{1}$ Rui Cruz Ferreira ${ }^{1}$

Hospital de Santa Marta, ${ }^{1}$ Lisboa - Portugal

\section{Resumo}

Fundamento: O tratamento com sacubitril-valsartana teve seu benefício prognóstico confirmado no ensaio PARADIGM-HF. No entanto, dados sobre alterações no teste de esforço cardiopulmonar (TECP) com o uso de sacubitril-valsartana são escassos.

Objetivo: O objetivo deste estudo foi comparar os parâmetros do TECP antes e depois do tratamento com sacubitril-valsartana.

Métodos: Avaliação prospectiva de pacientes com insuficiência cardíaca (IC) crônica e fração de ejeção do ventrículo esquerdo $\leq 40 \%$, mesmo sob terapia padrão otimizada, que iniciaram tratamento com sacubitril-valsartana, sem expectativa de tratamentos adicionais para a IC. Os dados do TECP foram coletados na semana anterior e 6 meses depois do tratamento com sacubitril-valsartana. Diferenças estatísticas com valor $p<0,05$ foram consideradas significativas.

Resultados: De 42 pacientes, 35 (83,3\%) completaram o seguimento de 6 meses, uma vez que 2 (4,8\%) morreram e $5(11,9 \%)$ interromperam o tratamento devido a eventos adversos. A média de idade foi de $58,6 \pm 11,1$ anos. A classe NYHA (classificação da New York Heart Association) melhorou em 26 (74,3\%) pacientes. O consumo máximo de oxigênio $\left(\mathrm{VO}_{2} \max \right)(14,4 \mathrm{vs} .18,3 \mathrm{ml} / \mathrm{kg} / \mathrm{min}, \mathrm{p}<0,001)$, a inclinação $\mathrm{VE} / \mathrm{VCO}_{2}(36,7 \mathrm{vs} .31,1, \mathrm{p}<0,001)$ e a duração do exercício (487,8 vs. 640,3 s, p<0,001) também melhoraram com o uso de sacubitril-valsartana. O benefício foi mantido mesmo com a dose de $24 / 26 \mathrm{mg}(13,5 \mathrm{vs} .19,2 \mathrm{ml} / \mathrm{kg} / \mathrm{min}, \mathrm{p}=0,018)$ de sacubitril-valsartana, desde que esta tenha sido a maior dose tolerada.

Conclusões: $\mathrm{O}$ tratamento com sacubitril-valsartana está associado a uma melhora acentuada do $\mathrm{VO}_{2}$ max, da inclinação VE/ $\mathrm{VCO}_{2}$ e da duração do exercício no TECP. (Arq Bras Cardiol. 2020; 115(5):821-827)

Palavras-chave: Insuficiência Cardíaca, Consumo de Oxigênio, Fração de Ejeção Ventricular, Ventilação Pulmonar, SacubitrilValsartana, Anti-Hipertensivos.

\section{Abstract}

Background: Sacubitril/valsartan had its prognosis benefit confirmed in the PARADIGM-HF trial. However, data on cardiopulmonary exercise testing (CPET) changes with sacubitril-valsartan therapy are scarce.

Objective: This study aimed to compare CPET parameters before and after sacubitril-valsartan therapy.

Methods: Prospective evaluation of chronic heart failure (HF) patients with left ventricular ejection fraction $\leq 40 \%$ despite optimized standard of care therapy, who started sacubitril-valsartan therapy, expecting no additional HF treatment. CPET data were gathered in the week before and 6 months after sacubitril-valsartan therapy. Statistical differences with a p-value $<0.05$ were considered significant.

Results: Out of 42 patients, 35 (83.3\%) completed the 6-month follow-up, since 2 (4.8\%) patients died and 5 (11.9\%) discontinued treatment for adverse events. Mean age was $58.6 \pm 11.1$ years. New York Heart Association class improved in 26 (74.3\%) patients. Maximal oxygen uptake $\left(\mathrm{VO}_{2} \max \right)(14.4$ vs. $18.3 \mathrm{ml} / \mathrm{kg} / \mathrm{min}, p<0.001), \mathrm{VE} / \mathrm{VCO}_{2}$ slope (36.7 vs. 31.1, $\left.p<0.001\right)$, and exercise duration $(487.8 \mathrm{vs} .640 .3 \mathrm{sec}, p<0.001)$ also improved with sacubitril-valsartan. Benefit was maintained even with the $24 / 26 \mathrm{mg}$ dose $(13.5 \mathrm{vs} .19 .2 \mathrm{ml} / \mathrm{kg} / \mathrm{min}, \mathrm{p}=0.018)$ of sacubitrilvalsartan, as long as this was the highest tolerated dose.

Conclusions: Sacubitril-valsartan therapy is associated with marked CPET improvement in $\mathrm{VO}_{2}$ max, VE/VCO slope, and exercise duration. (Arq Bras Cardiol. 2020; 115(5):821-827)

Keywords: Heart Failure; Oxygen Consumption; Stroke Volume; Pulmonary Ventilation; Sacubitril-Valsartan; Hypertension; Antihypertensive Agents Full texts in English - http://www.arquivosonline.com.br

Correspondência: António Valentim Gonçalves •

Hospital de Santa Marta - Rua de Santa Marta, 50. 1169-1024 Lisboa - Portugal

E-mail: antonio.a.goncalves.14@gmail.com

Artigo recebido em 08/07/2019, revisado em 21/09/2019, aceito em 23/10/2019

DOI: https://doi.org/10.36660/abc.20190443 


\section{Introdução}

O prognóstico de pacientes com insuficiência cardíaca (IC) mudou consideravelmente após a publicação de ensaios fundamentais (1987 - CONSENSUS, 1999 CIBIS-II ${ }^{2}$ e RALES ${ }^{3}$ ), que demonstraram o benefício do uso de antagonistas neuro-hormonais [inibidores da enzima conversora da angiotensina (IECA), betabloqueadores (BB) e antagonistas da aldosterona (AA), respectivamente] na melhora da sobrevida de pacientes com IC e na redução da fração de ejeção.

Vinte e sete anos depois do ensaio CONSENSUS, o ensaio PARADIGM-HF mostrou que o uso de sacubitrilvalsartana, uma combinação de inibidor da neprilisina e bloqueador do receptor da angiotensina II (BRA), poderia reduzir a hospitalização por IC e a mortalidade cardiovascular em $20 \%$ em comparação com o Enalapril. ${ }^{4}$

Como resultado, o sacubitril-valsartana recebeu uma recomendação de Classe I, nível de evidência B, como substituto do IECA em pacientes ambulatoriais com IC e fração de ejeção reduzida (ICFER) que permanecem sintomáticos mesmo sob tratamento ideal com IECA (ou BRA se o IECA não for tolerado), BB e AA. ${ }^{5}$ Contudo, o uso de sacubitril-valsartana não tem sido tão alto quanto esperado. ${ }^{6}$

Os objetivos do tratamento para pacientes com IC não são apenas prevenir a internação e reduzir a mortalidade, mas também melhorar o estado clínico e a capacidade funcional. O teste de esforço cardiopulmonar (TECP) é um forte preditor de mortalidade em pacientes com IC. Ele é utilizado como critério padrão para avaliar a necessidade de transplante cardíaco eletivo ${ }^{7}{ }^{7}$ sendo o consumo máximo de $\mathrm{O}_{2}\left(\mathrm{VO}_{2}\right.$ max $)$ e a relação entre ventilação e produção de $\mathrm{CO}_{2}$ (inclinação $\mathrm{VE} / \mathrm{VCO}_{2}$ ) as ferramentas de avaliação de risco mais adotadas. ${ }^{8}$

O volume de informação tem aumentado recentemente, já que alguns ensaios demonstraram melhora sintomática e funcional significativa após o início do tratamento com sacubitril-valsartana. ${ }^{9-12}$ Entretanto, o impacto na capacidade funcional requer pesquisas adicionais, pois a maioria dos ensaios tinha delineamento retrospectivo e, até onde sabemos, apenas um estudo prospectivo apresenta as alterações de parâmetros do TECP após o tratamento com sacubitril-valsartana. ${ }^{13}$

O objetivo deste estudo foi analisar prospectivamente a eficácia do tratamento com sacubitril-valsartana em uma coorte de pacientes com IC crônica sob terapia padrão otimizada, comparando os dados do TECP antes e depois do tratamento.

\section{Métodos}

A pesquisa foi conduzida em conformidade com os princípios estabelecidos na Declaração de Helsinque. O comitê de ética institucional e a Comissão Nacional de Proteção de Dados (CNPD, número de autorização 5962) aprovaram o protocolo do estudo.

Todos os pacientes forneceram consentimento livre e esclarecido por escrito.

\section{População de pacientes}

O estudo apresenta uma análise prospectiva de único centro realizada entre outubro de 2017 e junho de 2018.

Durante esse período, todos os pacientes ambulatoriais sob terapia padrão otimizada para IC crônica, com fração de ejeção do ventrículo esquerdo $\leq 40 \%$ e classe $\geq$ II na classificação da New York Heart Association (NYHA) foram orientados a iniciar o tratamento com sacubitril-valsartana de acordo com as diretrizes vigentes. ${ }^{5}$

\section{Definição de IC crônica sob terapia padrão otimizada}

A terapia padrão otimizada para IC crônica foi definida como mais de seis meses de tratamento com a dose máxima tolerada de um IECA ou BRA, conforme apropriado, um BB e um AA. O cardioversor desfibrilador implantável (CDI) e/ ou a terapia de ressincronização cardíaca (TRC) podem ser utilizados se indicados pelas diretrizes vigentes e se o paciente tiver sido adequadamente tratado de acordo com os padrões aplicáveis para doença coronariana e insuficiência mitral (IM) e não haja expectativa de alteração do tratamento adicional da IC nos 6 meses seguintes. Foram excluídos os pacientes que iniciaram um programa de exercícios nos 3 meses anteriores ou durante o tratamento com sacubitril-valsartana.

\section{Protocolo do estudo}

Todos os pacientes forneceram consentimento livre e esclarecido por escrito. Em seguida, foram obtidos dados clínicos, laboratoriais, de ecocardiograma transtorácico (ETT) e de TECP na semana anterior ao início do tratamento com sacubitril-valsartana.

Um período de eliminação (washout) de 36 horas permitiu a alteração de um IECA para o sacubitril-valsartana. O tratamento com sacubitril-valsartana foi preferencialmente iniciado com a dose de 49/51 mg duas vezes ao dia ou 24/26 mg duas vezes ao dia em pacientes com dose $<10 \mathrm{mg} /$ dia de Enalapril ou equivalente. Foram feitas tentativas de duplicar a dose a cada 2 a 4 semanas para atingir a dose-alvo de manutenção de 97/103 mg duas vezes ao dia, exceto em pacientes com pressão sistólica $<100 \mathrm{mmHg}$, hipotensão sintomática, hiperpotassemia $>5,5 \mathrm{mEq} / \mathrm{L}$ ou diminuição da taxa de filtração glomerular (TFG) para menos de $60 \mathrm{ml} / \mathrm{min}$, avaliada pela equação de Cockcroft-Gault.

Todos os pacientes foram acompanhados por seis meses a partir da data de conclusão dos exames e os dados clínicos, laboratoriais, de ETT e de TECP foram coletados novamente após seis meses de tratamento com sacubitril-valsartana.

O apêndice complementar fornece informações relativas a todos os dados levantados.

\section{Teste de esforço cardiopulmonar}

Um TECP máximo ou sintoma-limitado foi realizado em esteira utilizando o protocolo de Bruce modificado (esteira ergométrica GE Marquette Série 2000). A análise de gases foi precedida pela calibração do equipamento. A ventilação minuto, o consumo de oxigênio e a produção de dióxido de carbono foram obtidos respiração a respiração, por meio de um analisador de gases SensorMedics Vmax 
229. O VO max foi definido como a maior média de 30 segundos alcançada durante o exercício e foi normalizado para o índice de massa corporal. ${ }^{14} \mathrm{O}$ limiar anaeróbio (LA) foi determinado pela combinação de métodos padrão (inclinação $\mathrm{V}$ preferencialmente e equivalentes ventilatórios). A inclinação $\mathrm{VE} / \mathrm{VCO}_{2}$ foi calculada por regressão linear por mínimos quadrados, com base nos dados adquiridos ao longo de todo o exercício. Os pacientes foram incentivados a realizar o exercício até que a razão de troca respiratória (RTR) fosse $\geq 1,10$.

\section{Análise estatística}

As características iniciais são expressas em frequências (porcentagens) para variáveis categóricas e como médias e desvios padrão para variáveis contínuas. Todas as análises comparam os parâmetros dos pacientes no início e seis meses depois do tratamento com sacubitril-valsartana.

A distribuição normal das variáveis contínuas foi verificada pelo teste de Kolmogorov-Smirnov. O teste t para amostras pareadas comparou as variáveis antes e depois do tratamento com sacubitril-valsartana. Diferenças estatísticas com valor $p<0,05$ foram consideradas significativas. Os dados foram analisados no programa Statistical Package for the Social Science para Windows, versão 24.0 (SPSS Inc, Chicago, IL).

\section{Resultados}

\section{Visão geral da população estudada}

Um total de 42 pacientes participaram do estudo. Destes, $35(83,3 \%)$ completaram o seguimento de seis meses com sacubitril-valsartana, já que $2(4,8 \%)$ pacientes morreram (1 com hemorragia intracraniana pós-trauma e 1 com morte súbita cardíaca) e 5 (11,9\%) interromperam o tratamento devido a eventos adversos (2 com insuficiência renal aguda reversível e 3 com hipotensão sintomática com a menor dose de sacubitril-valsartana). Não houve perda de seguimento durante os seis meses.

A Tabela 1 apresenta as características iniciais dos 35 pacientes que completaram o seguimento de seis meses com sacubitril-valsartana. A média de idade foi de 58,6 $6 \pm 11,1$ anos, com 29 (82,9\%) pacientes do sexo masculino e etiologia isquêmica em 15 (42,9\%) participantes.

Esses pacientes eram altamente sintomáticos, como comprovado pela classe NYHA $\geq$ III em $51,4 \%$ deles e pelos $42,9 \%$ de hospitalizações por piora da IC no ano anterior ao tratamento com sacubitril-valsartana. Todos os pacientes usavam um IECA ou BRA associado a um BB e 94,3\% tomavam um AA. O CDI já estava implantado em 30 (85,6\%) pacientes, dos quais 7 (20,0\%) tinham o sistema TRC-D. Três (8,6\%) pacientes haviam sido previamente submetidos a um reparo percutâneo da valva mitral por meio do sistema de MitraClip®.

\section{Dose de sacubitril-valsartana}

O tratamento com sacubitril-valsartana foi iniciado com doses de 24/26 mg duas vezes ao dia em 18 (51,4\%) pacientes e 49/51 mg duas vezes ao dia em 17 (48,6\%) pacientes. Aos seis meses, a dose de $24 / 26 \mathrm{mg}$ duas vezes
Tabela 1 - Características iniciais da população do estudo $(n=35)$

\begin{tabular}{|c|c|}
\hline Características & $\mathrm{n}(\%)$ \\
\hline Média de idade (anos) & $58,60 \pm 11,14$ \\
\hline Etiologia isquêmica & $15(42,9 \%)$ \\
\hline Sexo masculino & $29(82,9 \%)$ \\
\hline$N Y H A \geq I I I$ & $18(51,4 \%)$ \\
\hline Média do índice de massa corporal $\left(\mathrm{kg} / \mathrm{m}^{2}\right)$ & $28,09 \pm 3,77$ \\
\hline Hospitalização por insuficiência cardíaca no ano anterior & $15(42,9 \%)$ \\
\hline Média de PNB (pg/ml) & $375,30 \pm 342,19$ \\
\hline Fumante & $7(20,0 \%)$ \\
\hline Hipertensão anterior & $25(71,4 \%)$ \\
\hline Dislipidemia & $25(71,4 \%)$ \\
\hline Diabetes mellitus & $11(31,4 \%)$ \\
\hline Doença arterial periférica & $4(11,4 \%)$ \\
\hline Histórico familiar de insuficiência cardíaca & $1(2,9 \%)$ \\
\hline Fibrilação atrial & $14(40 \%)$ \\
\hline Doença renal crônica & $2(5,7 \%)$ \\
\hline Doença hepática crônica & $0(0,0 \%)$ \\
\hline Inibidores da enzima conversora de angiotensina & $29(82,9 \%)$ \\
\hline Bloqueador do receptor de angiotensina II & $6(17,1 \%)$ \\
\hline Betabloqueadores & $35(100,0 \%)$ \\
\hline Antagonista da aldosterona & $33(94,3 \%)$ \\
\hline Ivabradina & $13(37,1 \%)$ \\
\hline Digoxina & $9(25,7 \%)$ \\
\hline Cardioversor desfibrilador implantável & $30(85,6 \%)$ \\
\hline Terapia de ressincronização cardíaca (TRC-D) & $7(20 \%)$ \\
\hline Reparo percutâneo da valva mitral com MitraClip $®$ & $3(8,6 \%)$ \\
\hline
\end{tabular}

NYHA: New York Heart Association; PNB: peptídeo natriurético tipo $B$.

ao dia era administrada a $10(28,6 \%)$ pacientes, 49/51 mg duas vezes ao dia a 11 (31,4\%) e 97/103 mg duas vezes ao dia a $14(40,0 \%)$.

Não foram encontradas alterações significativas quanto à dose expressa em porcentagem da dose-alvo de BB $(68,8$ $\pm 28,6 \%$ vs. $70,6 \pm 28,0 \%, p=0,278)$ e AA $(51,6 \pm 19,0 \%$ vs. $53,2 \% \pm 24,4 \%, p=0,352)$ ou à dose de diurético de alça expressa em equivalentes de furosemida (43,6 $\pm 27,6 \%$ vs. $39,1 \pm 26,5 \%, p=0,191)$ no início e após seis meses do tratamento com sacubitril-valsartana.

\section{Avaliação clínica}

Os 35 pacientes que completaram os seis meses de tratamento com sacubitril-valsartana apresentaram uma melhora relevante na classe NYHA, já que apenas $9(25,7 \%)$ deles permaneceram na mesma classe, enquanto 24 (68,6\%) melhoraram uma classe NYHA e $2(5,7 \%)$ melhoraram duas classes. Nenhum paciente teve piora da classe NYHA durante os seis meses de tratamento com sacubitril-valsartana e apenas $3(8,6 \%)$ permaneceram na classe III. 


\section{Avaliação do ecocardiograma transtorácico}

A Tabela 2 apresenta os resultados da análise do ETT. As dimensões do ventrículo esquerdo (VE) e os volumes atriais foram significativamente menores aos seis meses de tratamento. A excursão sistólica do anel tricúspide não apresentou diferenças significativa, independentemente da presença de diminuição na pressão sistólica da artéria pulmonar aos 6 meses de terapia. A fração de ejeção do ventrículo esquerdo teve elevação média absoluta de 5,9\%.

\section{Análise do TECP}

O tratamento com sacubitril-valsartana demonstrou um impacto substancial na capacidade funcional (Tabela 3). $\mathrm{O} \mathrm{VO}_{2}$ max, $\mathrm{O} \mathrm{VO}_{2}$ max previsto, a inclinação $\mathrm{VE} / \mathrm{VCO}_{2}$ e a duração do exercício tiveram uma melhora importante após o tratamento, sem diferença significativa no esforço do exercício, avaliado pela razão de troca. Não foram identificadas diferenças significativas relativas aos parâmetros de frequência cardíaca (FC) e pressão arterial.

A Tabela 4 fornece os parâmetros do TECP de acordo com a dose de sacubitril-valsartana. Os pacientes com doses de 24/26 mg e 49/51 mg aos 6 meses de tratamento com sacubitril-valsartana apresentaram o maior aumento nos valores de $\mathrm{VO}_{2}$ max e de inclinação $\mathrm{VE} / \mathrm{VCO}_{2}$.

Pacientes com IC tanto isquêmica $(16,9 \pm 7,1 \mathrm{ml} / \mathrm{kg} / \mathrm{min}$ vs. $20,2 \pm 4,2 \mathrm{ml} / \mathrm{kg} / \mathrm{min}, \mathrm{p}=0,014)$ quanto não-isquêmica $(12,6 \pm 4,6 \mathrm{ml} / \mathrm{kg} / \mathrm{min}$ vs. $17,0 \pm 5,1 \mathrm{ml} / \mathrm{kg} / \mathrm{min}, \mathrm{p}=0,004)$ demonstraram melhora no $\mathrm{VO}_{2}$ max aos 6 meses de tratamento com sacubitril-valsartana.

\section{Discussão}

O TECP é um forte preditor de mortalidade em pacientes com IC. Ele é utilizado como critério padrão para indicação de

Tabela 2 - Dados ecocardiográficos antes e seis meses depois do tratamento com sacubitril-valsartana

\begin{tabular}{lcc}
\hline & Tempo 0 & $\mathbf{6 ~ m e s e s ~}$ \\
\hline Dados Ecocardiográficos & & $\mathbf{p}$ \\
\hline Diâmetro diastólico final do ventrículo esquerdo $(\mathrm{mm})$ & $71,3 \pm 8,4$ & $66,9 \pm 7,6$ \\
\hline Diâmetro sistólico final do ventrículo esquerdo $(\mathrm{mm})$ & $57,8 \pm 9,4$ & $53,1 \pm 9,3$ \\
\hline Septo interventricular $(\mathrm{mm})$ & $9,6 \pm 1,7$ & $9,9 \pm 1,9$ \\
\hline Fração de ejeção do ventrículo esquerdo $(\%)$ & $29,3 \pm 6,4$ & $35,17 \pm 8,6$ \\
\hline Volume do átrio esquerdo $\left(\mathrm{m} / \mathrm{m}^{2}\right)$ & $51,5 \pm 22,6$ & $43,7 \pm 15,8$ \\
\hline Volume do átrio direito $\left(\mathrm{ml} / \mathrm{m}^{2}\right)$ & $33,1 \pm 4,4$ & 0,001 \\
\hline Pressão sistólica da artéria pulmonar $(\mathrm{mmHg})$ & $38,3 \pm 12,2$ & $28,5 \pm 13,5$ \\
\hline Excursão sistólica do anel tricúspide $(\mathrm{mm})$ & $19,2 \pm 4,4$ & $30,9 \pm 10,6$ \\
\hline
\end{tabular}

Valores são expressos como média \pm desvio padrão.

Tabela 3 - Dados do teste de esforço cardiopulmonar antes e seis meses depois do tratamento com sacubitril-valsartana

\begin{tabular}{|c|c|c|c|}
\hline & Tempo 0 & 6 meses & $\mathrm{p}$ \\
\hline \multicolumn{4}{|l|}{ Dados do teste de esforço cardiopulmonar } \\
\hline Frequência cardíaca máxima (bpm) & $114,1 \pm 27,2$ & $118,9 \pm 24,7$ & 0,110 \\
\hline Frequência cardíaca máxima prevista (\%) & $70,7 \pm 16,0$ & $73,9 \pm 14,7$ & 0,083 \\
\hline Recuperação da frequência cardíaca no primeiro minuto (bpm) & $17,0 \pm 12,3$ & $17,8 \pm 12,9$ & 0,720 \\
\hline Pressão arterial sistólica inicial (mmHg) & $115,8 \pm 18,3$ & $109,3 \pm 16,5$ & 0,094 \\
\hline Pressão arterial sistólica máxima (mmHg) & $140,0 \pm 29,8$ & $139,7 \pm 23,6$ & 0,946 \\
\hline Consumo máximo de oxigênio (ml/kg/min) & $14,4 \pm 6,0$ & $18,63 \pm 4,9$ & $<0,001$ \\
\hline Consumo máximo de oxigênio previsto (\%) & $49,6 \pm 18,7$ & $65,7 \pm 15,5$ & $<0,001$ \\
\hline Inclinação VE/VCO 2 & $36,7 \pm 7,2$ & $31,1 \pm 5,8$ & $<0,001$ \\
\hline Razão de troca respiratória de pico & $1,0 \pm 0,1$ & $1,0 \pm 0,1$ & 0,396 \\
\hline Duração do exercício até o LA (s) & $269,7 \pm 277,1$ & $292,5 \pm 253,2$ & 0,623 \\
\hline Consumo de oxigênio até o LA (ml/kg/min) & $12,0 \pm 4,3$ & $13,7 \pm 3,6$ & 0,087 \\
\hline
\end{tabular}

Valores são expressos como média \pm desvio padrão; LA: limiar anaeróbio. 
Tabela 4 - Dados do teste de esforço cardiopulmonar de acordo com a dose de sacubitril-valsartana

\begin{tabular}{llll}
\hline & Tempo 0 & 6 meses & p \\
\hline Dados do teste de esforço cardiopulmonar & & & \\
\hline Consumo máximo de oxigênio (ml/kg/min) & & $19,2 \pm 6,6$ & 0,018 \\
\hline Dose de 24/26 mg & $13,5 \pm 5,9$ & $17,6 \pm 4,3$ & 0,019 \\
\hline Dose de 49/51 mg & $13,5 \pm 6,6$ & $18,1 \pm 4,4$ & 0,085 \\
\hline Dose de 97/103 mg & $15,5 \pm 5,9$ & & 0,004 \\
\hline Consumo máximo de oxigênio previsto (\%) & & & 0,008 \\
\hline Dose de 24/26 mg & $44,9 \pm 20,6$ & $02,8 \pm 18,3$ & 0,048 \\
\hline Dose de 49/51 mg & $47,8 \pm 18,6$ & $69,1 \pm 14,1$ & \\
\hline Dose de 97/103 mg & $53,7 \pm 18,2$ & $65,2 \pm 15,6$ & \\
\hline Inclinação VE/VCO & & & 0,033 \\
\hline Dose de 24/26 mg & $38,0 \pm 8,9$ & $28,1 \pm 3,1$ & 0,005 \\
\hline Dose de 49/51 mg & $38,8 \pm 5,5$ & $31,9 \pm 3,0$ & 0,148 \\
\hline Dose de $97 / 103 \mathrm{mg}$ & $34,6 \pm 7,3$ & $32,0 \pm 7,7$ & \\
\hline
\end{tabular}

Valores são expressos como média \pm desvio padrão.

transplante cardíaco, ${ }^{7}$ sendo o $\mathrm{VO}_{2}$ max e a inclinação $\mathrm{VE} / \mathrm{VCO}_{2}$ os instrumentos de avaliação de risco mais utilizados. ${ }^{8}$ Vários tratamentos de IC (IECA, BB, AA, CDI, TRC) demonstraram melhorar a sobrevida dos pacientes e reduzir a fração de ejeção. A necessidade de revisar os critérios de priorização existentes para transplante cardíaco foi tema de debate, ${ }^{15}$ uma vez que o ensaio que definiu o uso de um ponto de corte $\leq 14 \mathrm{ml} / \mathrm{kg} / \mathrm{min}$ para o procedimento foi publicado antes de diversos avanços no tratamento da IC. ${ }^{16}$

Vários ensaios com BB não foram capazes de apresentar um aumento do $\mathrm{VO}_{2}$ max. ${ }^{17,18}$ Entretanto, o tratamento com BB aparentemente obteve um prognóstico melhor com o mesmo valor de $\mathrm{VO}_{2} \max _{,}{ }^{19,20}$ que foi utilizado para reduzir o ponto de corte para a seleção do transplante cardíaco de $14 \mathrm{ml} / \mathrm{kg} / \mathrm{min}$ para $12 \mathrm{ml} / \mathrm{kg} / \mathrm{min} .{ }^{21}$ Por outro lado, a TRC apresentou aumento da capacidade de exercício em um ensaio, com crescimento médio de $1,1 \mathrm{ml} / \mathrm{kg} / \mathrm{min}$ aos 6 meses, ${ }^{22}$ mas não conseguiu repetir o desempenho em outros ensaios. ${ }^{23,24}$

Com o objetivo de melhorar a capacidade funcional dos pacientes, alguns tratamentos recentes de $\mathrm{IC}$, como a modulação da contratilidade cardíaca, revelaram aumento do $\mathrm{VO}_{2}$ max de $0,65 \mathrm{ml} / \mathrm{kg} / \mathrm{min}^{25}$ para $0,84 \mathrm{ml} / \mathrm{kg} / \mathrm{min}^{26}$ aos 6 meses, enquanto o reparo percutâneo da insuficiência mitral secundária teve capacidade funcional preservada em um ensaio, avaliada pelo teste de caminhada de 6 minutos, ${ }^{27}$ mas não apresentou diferença em outro. ${ }^{28} \mathrm{O}$ treinamento com exercícios também mostrou melhora no $\mathrm{VO}_{2}$ max em ensaios anteriores, de 0,6 ml/ $\mathrm{min} / \mathrm{kg}$ aos 3 meses $^{29}$ a 2,1 ml/min/kg aos 2 meses. $^{30}$

Após o ensaio PARADIGM-HF confirmar que o tratamento com sacubitril-valsartana poderia reduzir a hospitalização por IC e a mortalidade cardiovascular em $20 \%$ em comparação com o Enalapril, ${ }^{4}$ o volume de informações tem aumentado, já que alguns ensaios revelaram melhora sintomática e funcional significativa depois do início do tratamento com sacubitril-valsartana. ${ }^{9-12}$ Contudo, a maioria dos ensaios tinha delineamento retrospectivo e, até onde sabemos, apenas um estudo prospectivo mostra as alterações de parâmetros do TECP após o tratamento com sacubitril-valsartana. ${ }^{13}$ Neste ensaio, com mediana de seguimento de 6 meses, o $\mathrm{VO}_{2}$ max aumentou em média 2,6 ml/min/kg e a inclinação VE/VCO, apresentou redução média de 2,4.

Nossos resultados mostram um grupo de pacientes com ICFER crônica altamente sintomáticos, como comprovado pela classe NYHA $\geq$ III em 51,4\% deles (apenas 23,9\% no ensaio PARADIGM-HF), pela pontuação inicial do Heart Failure Survival Score (HCSS) de 7,2 e pela taxa de hospitalização por piora da IC no ano anterior ao estudo de 42,9\%. Os pacientes recebiam terapia padrão otimizada, com percentual numericamente maior de indivíduos tratados inicialmente com BB $(100 \%$ vs. $93,1 \%)$, AA $(94,3 \%$ vs. $52,2 \%)$, CDI $(85,6 \%$ vs. $14,9 \%$ ) e TRC ( $20 \%$ vs. $7 \%$ ) em relação ao ensaio PARADIGM$\mathrm{HF}^{4} \mathrm{O}$ tratamento com sacubitril-valsartana foi iniciado com doses de 24/26 mg duas vezes ao dia em 18 (51,4\%) pacientes e 49/51 mg duas vezes ao dia em 17 (48,6\%) pacientes. Este procedimento é similar ao de um estudo recente de dados reais que iniciou o tratamento com sacubitril-valsartana com uma dose de 24/26 mg duas vezes ao dia em 51\% dos pacientes, 49/51 mg duas vezes ao dia em 38\% e 97/103 mg duas vezes ao dia em $11 \% .{ }^{31}$ No presente estudo, a dose média diária aos seis meses foi ligeiramente maior que a do estudo anterior (251 mg/dia vs. $207 \mathrm{mg} / \mathrm{dia})$, mas menor que a do ensaio PARADIGM-HF (375 mg/dia). ${ }^{4}$

Nessa população altamente sintomática, o tratamento com sacubitril-valsartana foi capaz de melhorar a classificação NYHA em pelo menos uma classe em $74,3 \%$ dos pacientes. Além da redução da classe NYHA, os dados do TECP demonstraram um aumento médio no $\mathrm{VO}_{2}$ max de $3,9 \mathrm{ml} / \mathrm{kg} / \mathrm{min}$ e uma redução média na inclinação $\mathrm{VE} / \mathrm{VCO}_{2}$ de 5,6, um valor numericamente maior que o benefício relatado anteriormente. ${ }^{13}$ Os valores mais elevados da fração de ejeção do ventrículo esquerdo e do 
VO max levaram a um crescimento significativo da $\operatorname{HFSS}(7,2$ $\pm 1,0$ vs. $7,9 \pm 0,9, p=0,001)$.

Esses resultados podem ser importantes ao se considerar pacientes que não estão sob tratamento com sacubitril-valsartana para transplante cardíaco com base nos valores do TECP, já que aos 6 meses de tratamento, o percentual de pacientes com $\mathrm{VO}_{2} \max \leq 12 \mathrm{ml} / \mathrm{min} / \mathrm{kg}$ diminuiu de 37 para $11 \%$ e com inclinação VE/NCO $>35$, de 52,4 a $17,1 \%$. Mais ensaios são necessários para verificar se os pontos de corte atuais para transplante cardíaco devem ser mantidos com o tratamento com sacubitril-valsartana.

Surpreendentemente, os pacientes com doses de 24/26 mg e 49/51 mg aos 6 meses de tratamento com sacubitril-valsartana apresentaram o maior aumento nos valores de $\mathrm{VO}_{2}$ max e inclinação $\mathrm{VE} / \mathrm{VCO}_{2}$, revelando o benefício desse tratamento desde que a maior dose tolerada seja administrada. Esses resultados podem complementar o contexto do uso de sacubitrilvalsartana na população com ICFER, pois a dose de 24/26 mg não foi usada no ensaio PARADIGM-HF.

Não é fácil explicar o aumento maior dos valores de $\mathrm{VO}_{2}$ max e inclinação $\mathrm{VE} / \mathrm{VCO}_{2}$ com a menor dose de sacubitril-valsartana. No entanto, esse cenário pode representar um viés, já que os pacientes que toleraram a maior dose de sacubitril-valsartana tinham valores iniciais de $\mathrm{VO}_{2}$ max elevados e valores baixos de inclinação $\mathrm{VE} / \mathrm{VCO}_{2}$, possivelmente tornando esse grupo menos propenso a ter um benefício maior com o tratamento.

\section{Limitações do estudo}

Este estudo tem limitações que devem ser referenciadas na interpretação dos resultados. Esta é uma experiência prospectiva de centro único; portanto, os achados podem refletir a prática local. Embora a amostra não seja grande, o estudo mostrou resultados promissores após apenas seis meses de tratamento, o que pode ser considerado uma motivação para aumentar o uso de sacubitril-valsartana em pacientes com tal indicação, conforme recomendam as diretrizes. ${ }^{5}$

Apesar de ser um estudo prospectivo, foram comparados os resultados iniciais e após seis meses de tratamento com sacubitril-valsartana sem um grupo controle que continuaria o tratamento com IECA ou BRA. Após os resultados do ensaio PARADIGM-HF, ${ }^{4}$ não seria ético deixar alguns dos pacientes sem um tratamento que demonstrou melhorar a sobrevida.

Uma estratégia para tentar reduzir o viés relacionado à melhora concomitante causada por outros tratamentos que não o com sacubitril-valsartana foi a escolha de pacientes para o estudo previamente sob terapia padrão otimizada (exceto o tratamento com sacubitril-valsartana) por mais de seis meses e que passaram por procedimento cardiovascular significativo não recente (implante de CDI ou TRC, procedimento de revascularização coronariana, tratamento valvar ou ablação por cateter de fibrilação atrial). Isso fica evidente por não haver diferenças na dosagem de BB e AA após seis meses de tratamento ou novo procedimento de revascularização coronariana, tratamento valvar ou ablação por cateter de fibrilação atrial.

\section{Conclusões}

O tratamento com sacubitril-valsartana aparentemente aumentou a capacidade funcional de pacientes com IC crônica, com melhora acentuada no $\mathrm{VO}_{2} \max , \mathrm{VO}_{2}$ max previsto, inclinação VE/VCO 2 e duração do exercício, bem como redução da classe NYHA. Esses resultados podem complementar o contexto do uso de sacubitril-valsartana na população com ICFER, mostrando manutenção do benefício mesmo com a menor dose do tratamento, desde que esta seja a maior dose tolerada.

\section{Contribuição dos autores}

Concepção e desenho da pesquisa: Gonçalves AV, Pereirada-Silva T, Galrinho AIVO, Soares R, Feliciano J, Ferreira RC; Obtenção de dados: Gonçalves AV, Pereira-da-Silva T, Galrinho AIVO, Rio P, Moreira RI, Silva S, Alves S; Análise e interpretação dos dados, Análise estatística e Redação do manuscrito: Gonçalves AV; Revisão crítica do manuscrito quanto ao conteúdo intelectual importante: Pereira-da-Silva T, Galrinho AIVO, Rio P, Soares R, Feliciano J, Ferreira RC.

\section{Potencial conflito de interesses}

Declaro não haver conflito de interesses pertinentes.

\section{Fontes de financiamento}

O presente estudo não teve fontes de financiamento externas.

\section{Vinculação acadêmica}

Não há vinculação deste estudo a programas de pósgraduação.

\section{Referências}

1. Group CTS. Effects of enalapril on mortality in severe congestive heart failure. Results of the Cooperative North Scandinavian Enalapril Survival Study (CONSENSUS). The New England journal of medicine. 1987;316(23):1429-35.

2. The Cardiac Insufficiency Bisoprolol Study II (CIBIS-II): a randomised trial. Lancet. 1999;353(9146):9-13.

3. Pitt B, Zannad F, Remme WJ, Cody R, Castaigne A, Perez A, et al. The effect of spironolactone on morbidity and mortality in patients with severe heart failure. Randomized Aldactone Evaluation Study Investigators. The New England journal of medicine. 1999;341(10):709-17.
4. McMurray JJ, Packer M, Desai AS, Gong J, Lefkowitz MP, Rizkala AR, et al Angiotensin-neprilysin inhibition versus enalapril in heart failure. The New England journal of medicine. 2014;371(11):993-1004.

5. Ponikowski P, Voors AA, Anker SD, Bueno H, Cleland JG, Coats AJ, et al. 2016 ESC Guidelines for the diagnosis and treatment of acute and chronic heart failure: The Task Force for the diagnosis and treatment of acute and chronic heart failure of the European Society of Cardiology (ESC). Developed with the special contribution of the Heart Failure Association (HFA) of the ESC. European journal of heart failure. 2016;18(8):891-975. 
6. Luo N, Fonarow GC, Lippmann SJ, Mi X, Heidenreich PA, Yancy CW, et al. Early Adoption of Sacubitril/Valsartan for Patients With Heart Failure With Reduced Ejection Fraction: Insights From Get With the Guidelines-Heart Failure (GWTG-HF). JACC Heart failure. 2017;5(4):305-9.

7. Mehra MR, Canter CE, Hannan MM, Semigran MJ, Uber PA, Baran DA, et al. The 2016 International Society for Heart Lung Transplantation listing criteria for heart transplantation: A 10-year update. The Journal of heart and lung transplantation : the official publication of the International Society for Heart Transplantation. 2016;35(1):1-23.

8. Butler J, Khadim G, Paul KM, Davis SF, Kronenberg MW, Chomsky DB, et al. Selection of patients for heart transplantation in the current era of heart failure therapy. Journal of the American College of Cardiology. 2004;43(5):787-93.

9. Sgorbini L, Rossetti A, Galati A. Sacubitril/Valsartan: Effect on Walking Test and Physical Capability. Cardiology. 2017;138 Suppl 1:17-20.

10. Chandra A, Lewis EF, Claggett BL, Desai AS, Packer M, Zile MR, et al. Effects of Sacubitril/Valsartan on Physical and Social Activity Limitations in Patients With Heart Failure: A Secondary Analysis of the PARADIGM-HF Trial. JAMA cardiology. 2018;3(6):498-505.

11. Beltran P, Palau P, Dominguez E, Faraudo M, Nunez E, Guri O, et al. Sacubitril/ valsartan and short-term changes in the 6-minute walk test: A pilot study. International journal of cardiology. 2018;252:136-9.

12. Lau CW, Martens P, Lambeets S, Dupont M, Mullens W. Effects of sacubitril/ valsartan on functional status and exercise capacity in real-world patients. Acta cardiologica. 2018:1-8.

13. Vitale G, Romano G, Di Franco A, Caccamo G, Nugara C, Ajello L, et al. Early Effects of Sacubitril/Valsartan on Exercise Tolerance in Patients with Heart Failure with Reduced Ejection Fraction. Journal of clinical medicine. $2019 ; 8(2)$.

14. Guazzi M, Arena R, Halle M, Piepoli MF, Myers J, Lavie CJ. 2016 Focused Update: Clinical Recommendations for Cardiopulmonary Exercise Testing Data Assessment in Specific Patient Populations. Circulation. 2016;133(24):e694-711.

15. Mehra MR, Kobashigawa J, Starling R, Russell S, Uber PA, Parameshwar J, et al. Listing criteria for heart transplantation: International Society for Heart and Lung Transplantation guidelines for the care of cardiac transplant candidates--2006. The Journal of heart and lung transplantation : the official publication of the International Society for Heart Transplantation. 2006;25(9):1024-42.

16. Mancini DM, Eisen H, Kussmaul W, Mull R, Edmunds LH, Jr., Wilson JR. Value of peak exercise oxygen consumption for optimal timing of cardiac transplantation in ambulatory patients with heart failure. Circulation. 1991;83(3):778-86.

17. Metra M, Giubbini R, Nodari S, Boldi E, Modena MG, Dei Cas L. Differential effects of beta-blockers in patients with heart failure: A prospective, randomized, double-blind comparison of the long-term effects of metoprolol versus carvedilol. Circulation. 2000;102(5):546-51.

18. Gullestad L, Manhenke C, Aarsland T, Skardal R, Fagertun H, Wikstrand $\mathrm{J}$, et al. Effect of metoprolol CR/XL on exercise tolerance in chronic heart failure - a substudy to the MERIT-HF trial. European journal of heart failure. 2001;3(4):463-8.
19. ZugckC, Haunstetter A, Kruger C, Kell R, SchellbergD, Kubler W, et al. Impact of beta-blocker treatment on the prognostic value of currently used risk predictors in congestive heart failure. Journal of the American College of Cardiology. 2002;39(10):1615-22.

20. Pohwani AL, Murali S, Mathier MM, Tokarczyk T, Kormos RL, McNamara DM, et al. Impact of beta-blocker therapy on functional capacity criteria for heart transplant listing. The Journal of heart and lung transplantation : the official publication of the International Society for Heart Transplantation. 2003;22(1):78-86.

21. Peterson LR, Schechtman KB, Ewald GA, Geltman EM, de las Fuentes L, Meyer $\mathrm{T}$, et al. Timing of cardiac transplantation in patients with heart failure receiving beta-adrenergic blockers. The Journal of heart and lung transplantation : the official publication of the International Society for Heart Transplantation. 2003;22(10):1141-8

22. Young JB, Abraham WT, Smith AL, Leon AR, Lieberman R, Wilkoff B, et al. Combined cardiac resynchronization and implantable cardioversion defibrillation in advanced chronic heart failure: the MIRACLE ICD Trial. Jama. 2003;289(20):2685-94

23. Abraham WT, Young JB, Leon AR, Adler S, Bank AJ, Hall SA, et al. Effects of cardiac resynchronization on disease progression in patients with left ventricular systolic dysfunction, an indication for an implantable cardioverterdefibrillator, and mildly symptomatic chronic heart failure. Circulation. 2004;110(18):2864-8.

24. De Marco T, Wolfel E, Feldman AM, Lowes B, Higginbotham MB, Ghali JK, et al. Impact of cardiac resynchronization therapy on exercise performance, functional capacity, and quality of life in systolic heart failure with QRS prolongation: COMPANION trial sub-study. Journal of cardiac failure. 2008;14(1):9-18.

25. Kadish A, Nademanee K, Volosin K, Krueger S, Neelagaru S, Raval N, et al. A randomized controlled trial evaluating the safety and efficacy of cardiac contractility modulation in advanced heart failure. American heart journal. 2011;161(2):329-37 e1-2.

26. Abraham WT, Kuck KH, Goldsmith RL, Lindenfeld J, Reddy VY, Carson PE, et al. A Randomized Controlled Trial to Evaluate the Safety and Efficacy of Cardiac Contractility Modulation. JACC Heart failure. 2018;6(10):874-83.

27. Stone GW, Lindenfeld J, Abraham WT, Kar S, Lim DS, Mishell JM, et al. Transcatheter Mitral-Valve Repair in Patients with Heart Failure. The New England journal of medicine. 2018;379(24):2307-18.

28. Obadia JF, Messika-Zeitoun D, Leurent G, lung B, Bonnet G, Piriou N, et al Percutaneous Repair or Medical Treatment for Secondary Mitral Regurgitation. The New England journal of medicine. 2018;379(24):2297-306.

29. O'Connor CM, Whellan DJ, Lee KL, Keteyian SJ, Cooper LS, Ellis SJ, etal. Efficacy and safety of exercise training in patients with chronic heartfailure: HF-ACTION randomized controlled trial. Jama. 2009;301(14):1439-50.

30. Karapolat H, Engin C, Eroglu M, Yagdi T, Zoghi M, Nalbantgil S, et al. Efficacy of the cardiac rehabilitation program in patients with end-stage heartfailure, heart transplant patients, and left ventricular assist device recipients. Transplantation proceedings. 2013;45(9):3381-5.

31. Martens P, Belien H, Dupont M, Mullens W. Insights into implementation of sacubitril/valsartan into clinical practice. ESC heart failure. 2018;5(3):275-83.

\section{* Material suplementar}

Para informação adicional, por favor, clique aqui. 